\title{
Las colonias francesas dentro de la ecología- mundo (1830-1962): el caso de los combustibles fósiles, los bosques y las plantaciones tropicales en Argelia y Vietnam
}

\section{Armel Campagne*}

\begin{abstract}
RESUMEN
Los imperios coloniales jugaron un papel importante en la historia del Capitaloceno a través de la apropiación y explotación de los recursos naturales baratos, de la mano de obra y las energías baratas del mundo extraeuropeo, pero también por su producción de las naturalezas capitalizadas dentro de él, y, más ampliamente su coproducción de un mundo ecológico caracterizado por intercambios económicos y ecológicos desiguales a escala global. Basado en una problemática resultante del trabajo de Jason Moore, una reinterpretación de la historiografía existente y un trabajo de archivo, este artículo muestra que, si bien es esclarecedor comprender la historia de los combustibles fósiles, los bosques y las plantaciones de Argelia (1830-1962) y de Vietnam (1858-1954) en términos de ecología-mundo, su contribución al desarrollo de la ecología-mundo debe examinarse a la luz de las especificidades de cada colonia y de cada sector.
\end{abstract}

En primer lugar, hay que matizar la hipótesis de una producción colonial de combustibles fósiles que sería sistemáticamente barata y destinada a los centros capitalistas occidentales de la ecologíamundo, no correspondiendo esta hipótesis ni al carbón argelino (caro y usado localmente) ni a los hidrocarburos argelinos (caros y exportados principalmente a Francia) ni al carbón vietnamita (barato pero exportado al sudeste asiático). Entonces, la historia conjunta de las plantaciones de caucho vietnamitas y la agricultura colonial argelina revela una capacidad desigual y nunca absoluta de los colonizadores franceses, siempre dependientes de las condiciones ecológicas locales, para crear naturalezas capitalizadas que generen productos tropicales baratos. Finalmente, el estudio combinado de la explotación colonial de los recursos forestales en Argelia y Vietnam permite mostrar hasta qué punto el ecologismo manifestado por los colonizadores franceses, en particular el de las administraciones forestales coloniales, no obstaculizó la producción. Para la ecología-mundo, este análisis, evidentemente, merecería ser profundizado por nuevos estudios de caso, pero constituye, sin embargo, un primer vistazo a la compleja inserción histórica de las colonias francesas en el mundo ecológico.

\section{Palabras Clave}

Historia; Colonialismo; Capitaloceno; Ecología-mundo; Imperio Colonial Francés.

\section{TitLE}

The French colonies within the world-ecology (1830-1962): the case of fossil fuels, forests and tropical plantations in Algeria and Vietnam

\section{Extended Abstract}

Colonial empires played a significant role in the history of the capitalocene through their appropriation and exploitation of nature, cheap labor and cheap energies in the extra-European world. In this way, colonial empires contributed to the creation of a world-ecology characterized by unequal economic and ecological exchanges at the global scale.This article, drawing on Jason Moore's conceptual framework, archival sources and the historical literature, takes as case studies the contribution to modern world-ecology of the two main colonies of the French colonial empire, Algeria and Vietnam, with their respective fossil, forest and agricultural resources. In the first part, the comparative analysis of fossil fuel productions in colonial Algeria and Vietnam aims to test the world-ecology hypothesis of a colonial production of energy characterized as systematically cheap and exported to western capitalist centers. In the second part, the joint history ofVietnam's rubber plantations and of

\section{DOI:}

https://doi.org// 0.15366/relacionesinternacionales2021.46.004

Formato de citación recomendado:

CAMPAGNE, Armel (2021). "Las colonias francesas dentro de la ecología-mundo (1830-1962): el caso de los combustibles fósiles, los bosques y las plantaciones tropicales en Argelia y Vietnam”, Relaciones Internacionales, $\mathrm{n}^{\circ}$ 46, pp. 63-80.

\section{*Armel} CAMPAGNE, Investigador $\mathrm{PhD}$ en el Instituto Universitario Europeo (EUI), con un trabajo de investigación sobre la historia de la minería del carbón en el Imperio colonial francés. Es autor de Le Capitalocène.Aux racines historiques du dérèglement climatique (Divergences, 2017),y de varios artículos sobre la historia ambiental del capitalismo y del colonialismo. Contacto: armel. campagne@eui.eu

Recibido:

I 5/09/2020

Aceptado:

04/I 2/2020

Traducción: Rami ZAHRAWI HAJ-YOUNES 
Algeria's settler agriculture seeks to determine to what extent colonizers succeeded in producing cheap tropical goods for western countries. Finally, in the third part, the combined study of the colonial exploitation of Vietnam and Algeria's forest resources intends to assess to what extent French colonial environmentalism interfered with the production of cheap wood exported to the world-ecology.

The first section of the article establishes that in the case of Algeria, the colonial production of fossil energy was not always cheap. In the case of coal, exploited in Kenadsa between 1917 and 1962, it was neither cheap nor exported to western capitalist centers due to its remote location, its high extraction and transportation costs, and its rebellious and scarce workforce, making it uncompetitive with cheaper British coals. In the case of oil and gas resources, although their exploitation from 1956 was profitable for private companies, and exported mainly to France until the 1970's, their price was higher than the Middle East's hydrocarbons. Moreover, their State-assisted development was undertaken due to strategic considerations ('energy security' and the possibility of paying oil in francs rather than in dollars) and despite the costs it implied - and not because it was cheap. This demonstrates that although peripheries of the capitalist world-ecology tend to produce cheap energy for the western centers, this is not always the case and can even by the opposite. The second section of the article shows that although Vietnamese coal was cheap and profitable to exploit for some - but not all - colonial companies, it was mainly exported to Asian countries. This indicates that cheap energy produced in colonies and based on unequal ecological exchange did not always benefit exclusively western capitalist centers. The third section of the article shows that French colonizers failed to create capitalist natures producing cheap tropical goods for western countries, only succeeding to cultivate similar agricultural goods (like wine) to those produced by western centers. This manifests that colonial agricultural productions can sometimes, despite the cheapness of the colonial workforce in both cases, be neither cheap nor environmentally complementary to those of western centers. However, colonial agriculture in Algeria did manage to bolster French settler colonialism economically, although it was costly for the metropolis through underproduction crises. The fourth section, by contrast, exhibits the successes of colonial scientists to acclimate hevea brasilianis to Vietnam and that of companies, with the assistance of the colonial administration, to produce cheap tropical raw materials for western industries. However, rubber plantations in Vietnam favored the spread of malaria amongst workers, a socio-ecological contradiction that was not too costly for plantations at first but that eventually led Vietnamese workers, frustrated by the absence of substantial reforms to better their life conditions, to revolt against plantations during the Indochina War (19461954). Hence, the success of French colonizers in Vietnam to create capitalist natures producing cheap tropical goods was far from absolute, with falling profits during the IndochinaWar due to the destruction of one tenth of rubber plantations. The fifth section of the article reveals that despite the environmentalist rhetoric that France had conquered Algeria to restore the alleged granary of Rome, the cheap appropriation and exploitation of Algerian forests by private colonial companies at the expense of local populations -especially that of cork trees- was not impeded by colonial environmentalism, but rather justified by it.This proves that, even in such a case, colonizers were more concerned with economic considerations than with ideological ones. Similarly, the environmentalist stance of the colonial forestry administration in Vietnam never obstructed the cheap exploitation of local forest resources by private colonial companies, but only justified the colonial appropriation of Vietnam's forests at the expense of local populations.

The comparative analysis of fossil fuel productions in colonial Algeria and Vietnam allows us to relativize and question the idea that colonial energy productions were systematically cheap and exported to western capitalist centers. Indeed, the importance of geo-strategic considerations can and did push colonial powers, in this case France, to fund at great cost unprofitable and/or uncompetitive energy productions in their colonies. The joint study of colonial Algeria's settler agriculture and Vietnam's rubber plantations provides evidence for the unequal capacity of colonizers to create capitalist natures producing cheap tropical goods. This indicates that colonizers were always to a certain extent dependent on local environmental conditions to create capitalist natures, although they did manage to significantly transform these conditions in both cases.This also shows that the development of colonial agricultural productions did not always have the same priorities as settler colonies; the colonial administration mainly aimed at the economic prosperity of settlers, even when their production competed with those of metropolitan farmers, while in non-settler colonies it rather encouraged the production of cheap tropical goods that were not producible in the colonial metropolis. Finally, the comparative history of the exploitation of forest resources in colonial Vietnam and Algeria by private companies shows that colonial environmentalism was not an obstacle to colonial deforestation, but rather justified the dispossession and blaming of local colonized populations. Hence, although the environmental preoccupations of some colonizers, linked with racial anxieties in Algeria and scientific forestry management principles in Vietnam, were sincere and sometimes clashed with the economic interests of private companies, they almost never prevailed over the latter.The world-ecology conceptual framework thus proves to be analytically useful for the study of French colonies and their productions, although it must be given greater nuance and complexity through the multiplication of case studies.

\section{KEYWORDS}

History; Colonialism; Capitalocene;World-ecology; French Colonial Empire. 


\section{ntroducción}

Los imperios coloniales jugaron un papel importante en la historia del Capitaloceno a través de la apropiación y explotación de naturalezas baratas, mano de obra barata y energías baratas del mundo extraeuropeo (Moore, 2016b), pero también a través de su producción de naturalezas capitalizadas (nature-in-capitalism) dentro de él (Moore, 20 I6b), y, más ampliamente, su coproducción de una ecología-mundo caracterizado por intercambios económicos y ecológicos desiguales a escala global (Bonneuil \& Fressoz, 2016; Campagne, 20I 7b; Malm, 20 I6b; Moore, 2007; Patel \& Moore, 2017). La contribución al desarrollo de esta ecología-mundo de combustibles fósiles, bosques y plantaciones en Argelia (1830-1962) y deVietnam (1858-1954), las dos principales colonias francesas de este período, aún no ha sido estudiada a pesar de su papel en el auge del capitalismo francés y global tanto en términos de suministro de materias primas y energía barata a las metrópolis imperiales y a la ecología-mundo (Bouchène et al., 2014; Brocheux \& Hémery, 20 I I; Marseille, 2005; Meynier, 20I 5; Murray, 1980; Saul, 2016). Sin embargo, este rol debe ser examinado sin dejar de lado ambigüedades (Brocheux \& Hémery, 20I I), costos y proteccionismo (Marseille, 2005), dlímites y tensiones (Cooper \& Stoler, 1997) y finalmente contradicciones sociológicas (Moore, 2015) del imperio colonial francés. Por tanto, se tratará de ver bajo qué modalidades es posible aplicar a las colonias francesas un análises en términos de ecología-mundo, y esto a partir de una reinterpretación de la literatura secundaria y de la investigación doctoral basada en fuentes primarias. El análisis comparativo de la producción de combustibles fósiles en Argelia y Vietnam (Campagne, 202I; Cantoni, 2017; Jaehyun, 2018; Murray, 1980; Saul, 2016) durante el período colonial permitirá contrastar la hipótesis de una producción colonial de combustibles fósiles que serían sistemáticamente baratas y destinadas a los centros capitalistas occidentales de la ecología-mundo mientras se estudian las raíces coloniales del cambio climático (Malm, 20l6b), un tema hasta ahora poco explorado para este que es el caso francés. La historia conjunta de las plantaciones de caucho vietnamitas (Aso, 2018; Bonneuil, 1997) y la agricultura colonial argelina (Clancy-Smith, 2016; Henni, 2020; Isnard, 1949) nos permitirá determinar en qué medida los colonizadores franceses logran crear naturalezas capitalizadas que generan productos tropicales baratos, imposibles de producir dentro de los centros capitalistas destinados a una ecologíamundo cad avez más dependiente de ellos y basada en una división ecológica de la producción agrícola. Finalmente, el estudio combinado de la explitación colonial de los recursos forestales en Argelia (Davis, 2007; Ford, 2008) y de (McElwee, 2016; Thomas, 2009) nos permitirá cuestionar hasta qué punto el ecologísmo colonial francés (Grove, 1997) se interpusieron en el camino de las producciones forestales coloniales baratas destinadas a un mundo ecológico que era cada vez más exigente con los productos forestales (Tucker \& Richards, 1983).

\section{El Imperio Colonial Francés ¿Un productor de combustibles fósiles baratos para una ecología-mundo?}

\section{I.I. La Argelia colonial, una excepción francesa en términos de combustibles fósiles baratos.}

La Argelia colonial, conquistada por Francia a partir de 1830, no disponía de fuentes de energía fósil conocidas en su territorio hasta una fecha avanzada, por lo que se vio obligada a importarla de la Francia metropolitana o del extranjero. (Meynier, 20I5).' De hecho, paradójicamente, la sociedad

Aparte de las obras citadas explícitamente, mis análisis sobre el carbón argelino y vietnamita se basan esencialmente en el trabajo de 
colonial compró carbón barato del Reino Unido en particular, lo que, gracias a la producción avanzada y la productividad laboral vinculada a la existencia centenaria de los imperativos de la competencia capitalista (Malm, 20l7), produjo carbón barato que exportó a destinos lejanos, hasta el punto de que incluso la marina francesa en el sudeste asiático dependió de él hasta la década de los noventa del siglo XIX (Fichter, 20I2, 20I9). El primer depósito de carbón argelino no se explotó así hasta 1917, precisamente por los problemas de suministro de carbón británico y el aumento de su precio - debido a la interrupción de las comunicaciones marítimas vinculadas al conflicto mundial (Meynier, 20I5). El depósito de Kenadsa, ubicado en la puerta de entrada al Sahara y en las fronteras del suroeste de Argelia, cerca de Colomb-Béchar y Marruecos, solo fue descubierto en esta fecha tardía debido a la tardía conquista de esta región (en el inicio de los años 1900) y una débil presencia francesa anteriormente debido a la hostilidad de las tribus locales (Frémeaux, 2010). Además, el yacimiento de Kenadsa sólo pudo ser explotado por el uso del ferrocarril como medio de conquista del suroeste argelino, ya que nunca habría tenido una razón de ser si no la tuviera. Línea Béchar - Orán. Por lo tanto, solo fue operado hasta la década de los treinta con el propósito de suministrar trenes en esta línea por parte de la estatal Algerian Railways Company. En efecto, al estar el carbón de Kenadsa ubicado en una zona desértica alejada de los centros de consumo, su extracción no podía estar motivada por necesidades locales distintas a las del ferrocarril colonial. Además, no resultaba nada rentable transportarlo a Orán, el primer centro importante de consumo de carbón cerca de Kenadsa, ya que esta localidad se encontraba a más de 700 kilómetros del ferrocarril y se abastecía de un carbón británico barato mucho más competitivo. Además, era de difícil extracción y de mala calidad, por lo que distaba mucho de ser una energía barata. Por último, la mano de obra local era muy escasa, nómada, dispersa y urebelde al trabajo subterráneo», lo que a pesar de los salarios muy bajos no la convertía realmente en una mano de obra barata desde la perspectiva del mercado. La empresa en términos de costos de contratación, debido a muy alta rotación y producción, debido a la baja productividad laboral.Así, si la apropiación del yacimiento de carbón de Kenadsa fue barata, dado que la administración colonial procedió de facto a una expropiación de sus propietarios habituales al considerar esta área como terra nullius, su explotación estuvo lejos de ser barata, y casi nunca fue rentable para el país. ferrocarriles estatales de la Argelia colonial. Precisamente por estos problemas financieros, algunos administradores coloniales consideraron a principios de la década de los años veinte, con el regreso de las importaciones británicas, confiar la mina a una empresa privada. La administración ferroviaria se opuso a esta decisión, creyendo que podría producir energía relativamente barata y competitiva aumentando el volumen de producción. Al final, por razones principalmente geoestratégicas, el Estado francés decidió que era necesario mantener este depósito en actividad y bajo el control de una administración pública en previsión de una nueva guerra, porque en caso de una nueva interrupción de las comunicaciones marítimas tendría que encargarse rápidamente de suministrar carbón a parte de Argelia. De hecho, si después de varios años de auge, la producción casi se detuvo a principios de la década de los treinta debido a la crisis económica mundial, se decidió mantener la extracción al mínimo en lugar de detenerla por completo, porque en anticipación al nuevo conflicto mundial requería la la mía esté preparada para una rápida expansión de su capacidad de producción, que sería imposible si dejara de mantenerse. Así, la producción de carbón de Kenadsa aumentó exponencialmente a partir de 1939, de unos pocos miles de toneladas extraídas anualmente a cientos de miles, y esto con la ayuda de trabajos

archivo dentro de los Archivos Nacionales de Ultramar Aix-en-Provence y, en lo que respecta al carbón vietnamita, también dentro de los Archivos Nacionales de Vietnam y los Archivos. du monde du travail de Roubaix. 
forzados en parte y todos racializados. La afluencia de mano de obra barata, en particular gracias al trabajo forzoso de extranjeros indeseables de Vichy (republicanos y anarquistas españoles, judíos, alemanes antinazis e italianos antifascistas, etc.) ciertamente no permitió la producción de energía barata, pero permitió satisfacer parte de las necesidades de carbón de la Argelia de Vichy y post Vichy. En teoría, incluso se suponía que permitiría la industrialización de Argelia y abastecería el ferrocarril transahariano de Argelia a Mali, incluso si estos dos proyectos se realizaron solo ligeramente, el segundo en particular debido a un entorno hostil. La mina también se mantuvo en actividad después de 1945 y se nacionalizó en 1946 precisamente porque iba a formar parte de un gran proyecto para el desarrollo económico y social de Argelia, que serviría de justificación para mantener a Argelia bajo supervisión. Éste continuó siendo explotado hasta 1962 principalmente por razones de estabilidad política regional, ya que nunca logró producir energía barata, en particular por la resistencia de los trabajadores y el consecuente aumento de los salarios tras su nacionalización y aplicación en Kenadsa de las leyes sociales y la estado de menores (Campagne, 202I; Larab, 2013). La mina se mantuvo así en actividad y se subvencionó masivamente incluso después del inicio de la explotación masiva de los depósitos de hidrocarburos argelinos (1956), ya que aunque ya no era útil desde el punto de vista energético, apoyaba a miles de personas que se temían únete al FLN maquis en caso de cierre. Por tanto, el carbón argelino se explotó desde 1917 hasta 1962 sin ser nunca barato, a pesar de los salarios muy bajos hasta 1946, debido a un entorno hostil y una mano de obra rebelde. Tampoco se exportó nunca y, por lo tanto, permaneció aislado de las redes de la ecología-mundo capitalista durante su explotación.

Los hidrocarburos argelinos explotados desde 1956, contrariamente a lo que se podría pensar, tampoco eran baratos, aunque muy rentables para las empresas privadas. (Saul, 2016). En efecto, la exploración sistemática del imperio colonial francés a partir de 1945 con miras a encontrar yacimientos de hidrocarburos no tenía para el Estado francés el principal objetivo de encontrar reservas de energía baratas, sino más bien volverse menos dependiente del petróleo barato de Oriente Medio, estaba fuera de la esfera de influencia francesa (Cantoni, 2017). Así, el deseo de independencia energética del Estado francés lo empujó a realizar considerables gastos de prospección en su imperio colonial, y fue solo a regañadientes que aceptó a principios de la década de los cincuenta la participación de compañías petroleras no francesas (pero no grandes) en la prospección. en Argelia porque estas empresas, a diferencia de las francesas, tenían las tecnologías necesarias para el rápido descubrimiento de yacimientos de petróleo y gas (Cantoni, 2017). Gracias a estas tecnologías, en 1956 se descubrieron los grandes yacimientos de petróleo y gas argelino, lo que permitió a Francia obtener energía francesa en grandes cantidades y a empresas privadas enormes ganancias (Cantoni, 2017; Saul, 2016). Sin embargo, los hidrocarburos argelinos eran más caros que los de Oriente Medio: por lo tanto, no fue por su naturaleza barata que fueron explotados, sino a pesar de su precio más alto, en particular porque salvaron a Francia de tener que deshacerse de sus preciadas reservas en dólares. Por supuesto, Argelia abastecerá a Francia a gran escala con combustibles fósiles hasta hoy, con un pico durante la década de los sesenta (Saul, 2016), pero no fue debido a su naturaleza particularmente barata que tuvo lugar este intercambio ecológicamente desigual entre una metrópoli colonial (entonces poscolonial) y su colonia (entonces ex colonia), incluso si la explotación de los hidrocarburos argelinos era muy rentable para los privados franceses. empresas como Saul ha demostrado (2016). Finalmente, las producciones de hidrocarburos de Argelia, precisamente por su falta de competitividad frente al petróleo barato de Oriente Medio, inicialmente permanecieron aisladas del mundo ecológico 
global, ya que eran casi exclusivamente exportadas a Francia (Saul, 20I6).

El caso de la Argelia colonial muestra así que la producción colonial de energía no siempre es barata y, en lo que al carbón se refiere, está destinada a los centros capitalistas occidentales del mundo ecológico, e incluso que a veces es menos barata y rentable que el de los centros capitalistas, y esto en particular cuando se trata de naturalezas alejadas de los centros capitalistas, con altos costos de transporte de energía y una mano de obra local recalcitrante y pequeña. Esto significa que hay una excepción importante a la ubicación de tendencia de la producción de energía barata dentro de las periferias de la ecología-mundo capitalista. (Malm, 20 I6a; Patel \& Moore, 2017): los imperativos geopolíticos y políticos de los centros capitalistas, que jugaron un papel particularmente importante en el desarrollo de la producción de combustibles fósiles en la Argelia colonial.

\section{I.2. El carbón del Vietnam colonial, una producción barata destinada a los países asiáticos}

El caso del desarrollo de la producción de combustibles fósiles en elVietnam colonial, conquistado entre 1858 y 1885 , es muy diferente al de Argelia. Por un lado, las producciones de carbón del norte de Vietnam a partir de la década de los noventa del siglo XIX resultaron, para dos de ellas, muy rentables, gracias a la mano de obra barata y a la ubicación cerca del Golfo de Tonkin que permitía exportar carbón a todo el sudeste asiático barato (Campagne, 2017, 2021; Jaehyun, 2018; Murray, 1980), lo que lo acerca a los ejemplos paradigmáticos de apropiación y explotación barata de Moore y Patel (2017). Por otro lado, a diferencia del petróleo y el gas de Argelia, la producción de carbón en el norte deVietnam casi nunca se ha utilizado para abastecer de energía a la metrópoli colonial, salvo unas pocas decenas de miles de toneladas de muy buen carbón en el país. Durante la década de los treinta (Campagne, 2017a, 202I; Jaehyun, 2018). Sin embargo, este contraste merece ser matizado en varios niveles. En primer lugar, algunas producciones de carbón en el norte y centro de Vietnam han sido grandes fracasos debido a un entorno que aumenta enormemente los costos de minería y transporte, entre otros (Campagne, 202I; Jaehyun, 20 I8). Entonces, el trabajo de los vietnamitas (y de los chinos) era ciertamente barato, pero sin embargo había mucha rotación y deserciones del trabajo, y una baja productividad del trabajo, como en la Argelia colonial, que no impidió ganancias significativas para unos raras empresas francesas, pero no hicieron de la explotación del carbón colonial en Vietnam un río largo y tranquilo, en particular debido a los frecuentes tifones (Campagne, 20I7a, 202I; Jaehyun, 20 I8). Además, si no hubo muchas exportaciones de carbón colonial a la metrópoli, las ganancias se repatriaron y una parte significativa del carbón vietnamita se utilizó para el desarrollo de industrias e infraestructura coloniales en el sur y norte de Vietnam (Campagne, 2017a, 202I; Jaehyun, 20I8). Finalmente, el carbón del norte de Vietnam no se explotó solo por razones económicas, ya que una de las razones de la conquista del norte y centro de Vietnam fue monopolizar sus recursos de carbón por razones geopolíticas, en este caso, el suministro de combustible para los buques de guerra franceses en el este de Asia (Campagne, 20I7a, 2020, 2021; Jaehyun, 20I8).

Sin embargo, no solo las diferencias en términos de energía y mano de obra barata siguen siendo indiscutibles entre las dos colonias francesas más importantes (Brocheux \& Hémery, 20 I I), pero también hay diferencias muy importantes en su respectivo registro en el mundo ecológico 
capitalista. De hecho, el carbón de Argelia se usó solo para usos de colonias internas, mientras que el deVietnam estaba conectado a las redes asiáticas de la ecología-mundo (Campagne, 20 I7a, 202I; Jaehyun, 2018). De hecho, desde los años noventa del siglo XIX hasta la de 1940, la mayor parte de la producción de carbón vietnamita se exportó desde los países de Asia oriental, debido al bajo precio del transporte marítimo (fuera de ciertos periodos de conflicto) en comparación con los costos del transporte terrestre (Campagne, 2017, 202I; Jaehyun, 2018).Así, la ecología del carbón chino y japonés, lejos de las costas marinas (Pomeranz, 2002) y tener que ser transportados en tren, hizo que sus producciones fueran menos baratas que las de Vietnam del Norte, de ahí una exportación masiva de carbón vietnamita a estos dos países desde la década de los veinte (Campagne, 2017a, 202I; Jaehyun, 2018). Capitalismo de Hong Kong (Hamilton, 2020) También se benefició de esta proximidad ecológica con el norte de Vietnam para su desarrollo industrial y urbano, así como el Imperio de Japón desde la década de los veinte y especialmente de 1940 a 1945 (Campagne, 2017a, 2021; Jaehyun, 2018).

El caso de la producción de combustibles fósiles en el Vietnam colonial muestra así que, si bien se trata de un caso clásico de intercambio ecológico desigual y de producción capitalista de energía barata con mano de obra barata, esta explotación no benefició, no principalmente a los centros capitalistas occidentales de desde el punto de vista energético,y esto además precisamente por razones de rentabilidad de las propias empresas coloniales.

\section{I.3. Conclusión}

La comparación de la producción de hidrocarburos argelinos, carbón vietnamita y carbón argelino permite relativizar y complicar la idea de producción sistemáticamente barata de combustibles fósiles destinada a los centros capitalistas occidentales del mundo ecológico. El carbón vietnamita encaja bastante bien en este paradigma, pero esto no debería enmascarar una gran disparidad en el éxito económico entre las operaciones de carbón vietnamitas y sus dificultades en términos de mano de obra. El carbón argelino constituye por el contrario un caso límite, una excepción que confirma la regla, demostrando la importancia de los factores geopolíticos en la producción de combustibles fósiles, y esto hasta hoy con el esquisto y los hidrocarburos bituminosos, por muy lejos que sea "barato de producir en el empezar y en cualquier caso muy poco competitivo económicamente. El caso de los hidrocarburos argelinos se encuentra entre los dos escenarios, ya que por un lado su producción se ha desarrollado sobre todo por razones geopolíticas y a pesar de los importantes costes de prospección y producción, pero por otro lado esta producción ha demostrado ser rentable para empresas privadas. En cualquier caso, esto demuestra la importancia del estado y los imperativos geopolíticos en la producción de combustibles fósiles, que por lo tanto no necesariamente son baratos, sabiendo que rara vez lo es desde el punto de vista del estado por los costos de desarrollo. la infraestructura necesaria para la circulación de combustibles fósiles. 


\section{La creación en el Imperio Colonial Francés de naturalezas capitalizadas que generan productos tropicales baratos: entre fracasos y éxitos}

\section{I. La Argelia colonial, del fracaso de las naturalezas planificadas tropicales con la expansión de producciones agrícolas no complementarias y no competitivas}

La creación de naturalezas capitalizadas generando productos tropicales baratos, económicamente remunerativos y ecológicamente imposibles de producir dentro de los centros capitalistas debido a su clima, fue uno de los principales objetivos de los diversos imperios coloniales como ha demostrado Jason Moore (2007). Esta creación, cuando tuvo éxito, permitió crear nuevas oportunidades de lucro y satisfacer una demanda existente de productos tropicales dentro del mundo ecológico (o incluso crearla), sin correr el riesgo de competir con las producciones de las metrópolis imperiales, estableciendo así una división ecológica de las producciones agrícolas dentro de la ecología-mundo. Por todas estas razones,Argelia fue, a pesar de su clima mediterráneo, fantaseada por los viajeros de principios del siglo XIX, luego colonizadores desde 1830-1840, como un sitio potencial para el desarrollo de cultivos tropicales comerciales (Clancy-Smith, 2016), además de ser visto como el antiguo granero de Roma (Davis, 2007). Sin embargo, Argelia era ecológicamente inadecuada para la siembra de cultivos tropicales debido al «enfriamiento nocturno frecuente, prolongado y severo, fatal para las plantas tropicales» (Isnard, 1949, p. 2I3). También se caracterizó por la falta de recursos hídricos, suelos frágiles y frecuentes sequías (Clancy-Smith, 2016). Sin embargo, como dice el geógrafo Isnard, «durante mucho tiempo tuvimos la ilusión de desarrollar el cultivo a gran escala de productos tropicales en la nueva posesión» (Isnard, 1949, p. 212). El agrónomo Loiseleur-Deslonchamps, reportero de proyectos culturales en la Sociedad Colonial de Argel en 1832, incluso pensó que podríamos compensar la pérdida de la isla azucarera de Saint-Domingue (Haití) en I 804 con plantaciones de caña de azúcar, y que el el cultivo del café enriquecería a los colonos establecidos en las montañas argelinas (Isnard, 1949). El entonces gobernador general de Argelia, el mariscal Bertrand Clauzel, se sintió atraído por estas perspectivas y declaró que «todo tipo de culturas que enriquezcan nuestras colonias se pueden abordar y continuar con éxito en Argelsegún él, “la caña de azúcar, el algodón, el café prosperarán allí por sí solos; allí se obtiene fácilmente el cacao, y el índigo, cultivado con mimo, se aclimatará allí en poco tiempo» (Isnard, 1949, p. 212). Con esto en mente, rápidamente se creó un jardín de aclimatación en Argel, el Jardin d'Essai, pero el cultivo de caña de azúcar, café y cacao nunca pasó de la etapa experimental (Isnard, 1949), demostrar la incapacidad de las ideas para convertirse en fuerzas materiales en algunos casos. Sin embargo, las plantaciones de algodón, particularmente en la región de Orán, se multiplicaron entre 186I y 1865 gracias a la interrupción parcial de las importaciones de algodón de Estados Unidos vinculadas a la Guerra Civil (Isnard, 1949). El plantador y político Borély de la Sapie declaró que «el algodón es la planta que debe popularizar Argelia, que debe enriquecerla y unirla para siempre con Francia» (Isnard, 1949, p. 2 I 2). Sin embargo, esta euforia terminó con el restablecimiento de las importaciones estadounidenses, lo que provocó la casi total desaparición de las plantaciones de algodón en pocos años (Isnard, 1949), aunque persistieron durante toda la época colonial.

El fracaso de estas naturalezas planificadas (Moore, 2015) tropicales, al igual que el cultivo del tabaco y el lino, finalmente obligó a los colonos a recurrir a los cultivos de cereales (Isnard, 1949), menos lucrativo pero aclimatado a Argelia, que exportó su trigo a Francia desde 1730 hasta 
la primera década del siglo XIX (Touati, 20I8). Pero incluso las fantasías de restaurar el llamado granero de Roma experimentaron su parte de fracasos a lo largo del siglo XIX, debido a naturalezas planificadas desconectadas de las realidades ecológicas locales, como los muchos proyectos de cultivo de céréales (Clancy-Smith, 2016). La idea del mariscal Bugeaud de una conquista de Argelia a espada y arado (Sessions, 20I4) no se materializó finalmente hasta finales del siglo XIX, gracias a la masiva inversión estatal en riego (Clancy-Smith, 2016). Argelia se convirtió así hasta la década de los treinta en un exportador neto de trigo a Francia, importando luego parte de este trigo en forma de harina para no competir con los molinos harineros metropolitanos (Henni, 2020). Sin embargo, la Argelia colonial nunca se convirtió en el granero de la nueva Roma imperial, ya que su producción alcanzó como máximo un poco más de una quinta parte de la de la metrópoli a mediados de la década de los cincuenta, e incluso se convirtió en importador neto de trigo metropolitano de 1940 (Henni, 2020). Además, incluso cuando Argelia era un exportador neto de trigo a Francia, su producción, si permitía reducir la escala de las importaciones metropolitanas de trigo extranjero (Francia solo se volvió autosuficiente en trigo después de los años sesenta), no fue particularmente barato ya que era más caro excluyendo los derechos de aduana que el de los Estados Unidos (Henni, 2020). Finalmente, desde el punto de vista ecológico, constituyó una simple extensión de la producción metropolitana y no, como se concibió durante las primeras décadas de la colonización, una producción ecológicamente distinta y, por tanto, complementaria a esta última en la ecología-mundo.

La viticultura argelina, que representó el 53\% del valor de las exportaciones argelinas en 1938 gracias a una rentabilidad por hectárea 10 veces mayor que la de los cereales (Isnard, 1949), es un ejemplo similar de una simple extensión ecológica de la producción agrícola metropolitana. Es un intercambio colombiano (Crosby, 2003) tardío, que brindará la oportunidad para el rápido desarrollo de un monocultivo de vino en Argelia: una invasión de insectos de la costa este de Estados Unidos, que provocará la crisis de la filoxera, una enfermedad que destruirá en unos años casi todas las cepas metropolitanas, provocando una guerra de treinta años durante la cual la producción metropolitana, se relanzó paulatinamente de portainjertos resistentes de los Estados Unidos, seguirá siendo limitado (Garrier, 1989). Por lo tanto, la necesidad ecológica de un resurgimiento del capitalismo vitivinícola francés desde una frontera de recursos (Moore, 2015) colonial impulsará una colonización ecológica de Argelia por las vides. Los defensores de una Argelia tropical se habían opuesto hasta entonces al desarrollo de un viñedo en Argelia para no competir con las vides metropolitanas (Isnard, 1949). La falta de fondos también había bloqueado el desarrollo de las vides, hasta que el gobierno francés obligó a la Banque de l'Algérie a financiar generosamente a los inversores en vino, la gran mayoría de los cuales eran blancos (Henni, 2020) a partir de 1880, fecha en la que se animó a los colonos a desarrollar el cultivo de la vid en Argelia a causa de la crisis ecológica del viñedo francés (Isnard, 1949).Así, como afirma Isnard, «se forjó el instrumento más eficaz para la colonización deArgelia» (Isnard, 1949, p. 216 permitiendo finalmente que la agricultura colonial se desarrolle de manera rentable, provocando una transferencia masiva de tierra en beneficio de los colonos y en detrimento de los colonizados (Henni, 2020). En dos décadas, gracias al abundante crédito, la tierra barata, los altos precios, la mano de obra barata y un mercado metropolitano casi reservado como resultado de aranceles proteccionistas, la viticultura colonial de Argelia se convertirá en la primera fuente de exportación argelina y la principal fuente de importación de vino a la metrópolis (Henni, 2020), incluso si fue solo su bodega por un corto tiempo. La colonia se vuelve entonces completamente dependiente de la viticultura 
no solo económicamente, sino también política y socialmente, ya que asegura la presencia masiva de colonos europeos en el campo argelino. (Henni, 2020). Como resultado, nunca se cuestionará fundamentalmente durante el período colonial a pesar de la oposición de los viticultores metropolitanos, los recortes temporales de precios y las crisis regulares de sobreproducción. (Henni, 2020). Argelia finalmente se convirtió en el cuarto productor de vinos más grande del mundo, y su viticultura constituye un ejemplo arquetípico de naturalezas capitalizadas creadas con los medios, en particular, de una mano de obra barata sobreexplotada (Henni, 2020), su producción no aseguró una producción ecológicamente distinta y por tanto complementaria a la de los centros capitalistas dentro de la ecología-mundo.Además, estaba destinado solo al mercado metropolitano y, por lo tanto, en gran medida desconectado de la ecología-mundo. Además, no era particularmente barato de comprar, y fue solo por las medidas proteccionistas que no fue eliminado debido a su falta de competitividad (Henni, 2020).

En conclusión, el caso de Argelia constituye un ejemplo paradigmático del fracaso en la creación de naturalezas capitalizadas que proporcionen producciones baratas o ecológicamente distintas de las de las naturalezas capitalizadas metropolitanas, sin que se produzca un complemento ecológico entre metrópolis y colonia en la ecología-mundo, ni a una producción colonial de alimentos no específicos pero baratos, y esto a pesar de una mano de obra colonial barata. Sin embargo, estas naturalezas capitalizadas, en gran parte desconectadas del mundo ecológico debido al proteccionismo aduanero, permitieron que el colonialismo de colonos en Argelia fuera económicamente sostenible, aunque no sin inconvenientes a largo plazo desde el punto de vista metropolitano, fuera del período de filoxera y periodos de crisis de la ecología cerealista mundial y aumento del precio del trigo en la importación. Precisamente porque Argelia era una colonia de asentamiento con un clima mediterráneo, se desarrolló de una manera diferente a las colonias de explotación que producían bienes baratos y ecológicamente distintos de las producciones metropolitanas, por lo tanto un lugar muy diferente. Producciones agrícolas de Argelia y otras colonias francesas dentro de la ecología-mundo.

\subsection{Las plantaciones de caucho en el Vietnam colonial, naturalezas capitalizadas que producen ganancias, epidemias y revueltas}

La principal transformación ecológica del Vietnam colonial, con un importante legado poscolonial, fue probablemente el auge de las plantaciones de caucho desde principios del siglo XX (Aso, 20 I 8), aunque también hubo un desarrollo significativo de las plantaciones de té, café y pimienta (Brocheux \& Hémery, 20I I). La hevea brasilianis fue domesticada por un científico suizo en Vietnam a finales del siglo XIX, antes de extenderse bajo el impulso de la administración colonial y especialmente de las empresas metropolitanas en las tierras grises (alrededor de Saigón) ya las tierras rojas (más cercanas a Camboya) del sur deVietnam, transformando áreas ecológicamente diversas en monocultivos de árboles de caucho (Aso, 2018). Las plantaciones de caucho constituyen así un ejemplo arquetípico de conocimientos-poderes que han hecho posible crear naturalezas capitalizadas por la acción conjunta de científicos, administradores coloniales y capitalistas (Moore, 20I5), resultando en una disciplinarización de los trópicos según una lógica capitalista (Bonneuil, 1997). La expansión de estas naturalezas capitalizadas estuvo ligada a nuevas oportunidades de lucro tras la explosión de las necesidades de caucho de la ecología-mundo debido al desarrollo del automóvil a principios del siglo XX (Aso, 20l8). Si la expansión de las plantaciones de caucho es en parte de un proceso 
más amplio de apropiación barata de la naturaleza de Vietnam ya mencionado anteriormente, fue más allá en el sentido de que no explotó los recursos existentes, sino que los creó desde cero, generando ganancias colosales (Aso, 20 I8; Brocheux \& Hémery, 20 I I). La administración colonial alentó la expansión de la tierra de las empresas de plantaciones, principalmente en manos de colonos y sociedades metropolitanas, vendiéndoles tierras grises a precios moderados, adecuadas a expensas de las sociedades campesinas locales, o otorgándoles concesiones gratuitas. a expensas de las sociedades aborígenes de la montaña, imponiéndoles pocas restricciones normativas gracias a un marco de tenencia de la tierra muy liberal (Aso, 2018). Sin embargo, esta expansión barata a expensas de las poblaciones locales, ya que fueron desalojadas de las plantaciones donde habían vivido anteriormente en algunos casos, no estuvo exenta de conflictos, lo que provocó problemas recurrentes de seguridad en las plantaciones (Aso, 2018) recuerda a las minas de carbón de la primera década del siglo XIX (Campagne, 202I; Jaehyun, 20I8). Los Montagnards eran vistos no sólo como bandidosque amenazaban las plantaciones con sus incursiones, sino también como bárbaros con sus prácticas de tala y quema que supuestamente amenazaban las plantaciones (Aso, 20 18), a pesar de que estos, cuando fueron creados, habían involucrado limpiar la tierra con fuego (Bonneuil, 1997). Asegurar las plantaciones, por lo tanto, requería el control de las poblaciones forestales, la instalación de guardias nativos y comisarías de policía alrededor de las plantaciones y, finalmente, cuando las plantaciones no eran contiguas a cursos de agua, por la constitución una red de caminos coloniales que permitieran una conexión rápida y segura con las redes de la ecología-mundo (Aso, 2018).Así, la disciplinarización de los trópicos y sus habitantes a menudo fue de la mano, aunque nunca fue completa y generó sus propias contradicciones ecológicas internas, en particular con la expansión industrial de la malaria debido a la naturaleza capitalizada muy favorable a la reproducción del portador. mosquitos, Anopheles, presentes anteriormente pero en menor cantidad (Aso, 2018). Sin embargo, las empresas de las plantaciones prestaron poca atención a poner fin a las masacres de trabajadores vinculados a la malaria hasta la década de los treinta, contentándose con abandonar temporalmente algunos sitios particularmente infestados, y esto a pesar de los importantes costos de rotación (Aso, 2018). Sin lugar a dudas, la afluencia regular de mano de obra barata del delta del río Rojo permitió a las empresas de plantaciones escapar de los costos de la biopolítica preventiva y curativa (Aso, 2018). Sin embargo, esta falta de prevención diezmó las sucesivas oleadas de migrantes cuyos sistemas inmunológicos se vieron debilitados por un largo viaje en condiciones precarias y agrupados en hábitats insalubres y desprotegidos (Aso, 2018). Así, esta mano de obra con salarios muy bajos y condiciones de vida muy duras, cercana a la de las minas de carbón del norte (Campagne, 2017, 2021; Jaehyun, 2018; Murray, I 980), tuvo que sufrir además el producto de naturalezas capitalizadas particularmente letales (Aso, 20I8). Los trabajadores de las plantaciones protestaron contra esta doble opresión del capitalismo en la naturaleza y las naturalezas capitalizadas, pero fue solo cuando este estado de salud se volvió económicamente amenazador que algunas zonas establecieron una biopolítica paternalista, con mejoras sanitarias en términos de hábitat y mosquitos, redes y medidas de ingeniería ecológica como un mejor drenaje del agua, y autoritarias, con distribuciones forzadas de quinina, hasta entonces producidas en cantidades insuficientes y poco distribuidas entre los trabajadores vietnamitas (Aso, 2018). La mayoría de los plantadores, sin embargo, no estaban dispuestos a asumir el costo de tal biopolítica, prefiriendo dejar la carga financiera de la infraestructura médica y de salud a la administración colonial (Aso, 20 I8).Además, atribuyeron la hecatombe ligada a la malaria a una mayor vulnerabilidad racial de los migrantes del Delta del Río Rojo en comparación con los Montagnards considerados más resistentes, de ahí una división racializada del trabajo basada en 
criterios ecológicos (Aso, 20l8). Así, las empresas caucheras favorecieron la epidemia de malaria mientras se negaban a asumir el costo de una biopolítica preventiva y curativa, prefiriendo dejar este cuidado a la administración colonial, e incluso negándose a reconocer su responsabilidad por una pseudoecología racial.

Desde la década de los noventa del siglo XIX en adelante, las plantaciones se enfrentaron rápidamente a una escasez de mano de obra, lo que resultó en intentos esporádicos de contratar mano de obra extranjera, antes de finalmente recurrir a la mano de obra del delta del río Rojo (Aso, 20 18). Este último, obligado a realizar un trabajo asalariado debido a las adversas condiciones económicas del Delta del Río Rojo creado en parte por la colonización (Aso, 20I8; Brocheux \& Hémery, 20I I; Campagne, 2017a, 202I; Jaehyun, 20 I8; Murray, 1980), y atraída por falsas promesas de ganancias económicas, todavía era probable que abandonara las plantaciones una vez que descubriera las condiciones de vida y de trabajo, y en particular la extensión de la epidemia de malaria, que prevalecía sobre ellas, de ahí una deserción laboral muy importante (Aso, 2018) que también se encontró en las minas de carbón del norte (Campagne, 2017, 2021; Jaehyun, 2018; Murray, 1980). Por lo tanto, las empresas de las plantaciones presionaron a la administración colonial para que hiciera el contrato de trabajo lo más largo y restrictivo posible, lo que resultó en el código laboral de 1918 que vinculaba legalmente a los trabajadores con sus empleadores por un período de tiempo de al menos un año y provocó la deserción de plantaciones punibles con multas importantes o incluso penas de prisión (Aso, 2018). Sin embargo, las empresas de plantaciones en ocasiones fueron más allá de este marco legal al organizar un sistema de endeudamiento y vigilancia y traer desertores por la fuerza y dándoles un trato extralegal (Aso, 2018). Y las disposiciones favorables a los empleados, especialmente en términos de condiciones sanitarias, solo se observaron en raras ocasiones (Aso, 2018).Así, lejos de fortalecer a los cuerpos vietnamitas, las plantaciones se capitalizaron de naturalezas altamente peligrosas para ellos, debido a la malaria, el berberi (deficiencia de vitamina $\mathrm{BI}$ ) y las condiciones de migración, trabajo y vivienda (Aso, 2018). La magnitud de este desastre sanitario, revelada por la prensa, obligó al gobierno colonial a promulgar un nuevo código laboral en 1927 (Boucheret, 2008), más protector en términos de salud pero que tendría poco efecto concreto (Aso, 2018). Los colonos también consideraron que los trabajadores eran responsables de su estado de salud por perder sus raciones de comida en París, por ser adictos al opio, por no tomar sus dosis de quinina o por negligencia e ignorancia (Aso, 2018). Y cuando existían instalaciones sanitarias privadas, lo cual era raro ya que los hacendados consideraban que la administración colonial era la responsable del campo sanitario, el cuidado era más que sumario (Aso, 20 I8). El fracaso de las reformas coloniales, incluidas las del Frente Popular, y la falta de mejoras significativas en las condiciones de vida y de trabajo en las plantaciones fomentaron la difusión de ideas anticolonialistas y una radicalización de las luchas obreras (Aso, 2018). Por lo tanto, no es sorprendente que los trabajadores de las plantaciones de caucho desempeñaran un papel destacado durante la Guerra de Indochina de 1946-1954 junto con el Vietminh, que a su vez hizo de las plantaciones el símbolo mismo de la explotación colonial (Aso, 2018). Las plantaciones se convirtieron entonces en un campo de batalla real y simbólico entre los hacendados y el ejército francés por un lado y los trabajadores vietnamitas y el Vietminh por el otro, este último partiendo de una política de tierra arrasada destinada a arruinar la economía colonial mediante la destrucción de las plantaciones y su equipo a un enfoque que considera estas plantaciones como recursos nacionales (futuros) que deben ser capturados y explotados en beneficio de la nación vietnamita, asegurando su sostenibilidad 
en la era socialista (Aso, 20I8). Sin embargo, mientras tanto, más del 10\% de estas naturalezas capitalizadas fueron destruidas en una forma de ludismo anticolonial, con un impacto significativo en el nivel de ganancias de las plantaciones y las exportaciones de caucho barato al mundo ecológico (Aso, 2018).

Las plantaciones de caucho del sur de Vietnam representan así un ejemplo de una exitosa creación ex nihilo de naturalezas capitalizadas por la alianza de científicos, administradores coloniales y capitalistas. Sin embargo, estas naturalezas capitalizadas favorecieron una epidemia de malaria que hizo de las plantaciones de verdaderas cárceles sanitarias, y ante la ausencia de una biopolítica, en particular antipalúdica, mejorando significativamente sus condiciones de vida y de trabajo, los trabajadores vietnamitas intentaron desertar masivamente, luego obtener reformas, antes de atacar directamente las naturalezas capitalizadas. De hecho, las contradicciones socio-ecológicas de las plantaciones de caucho terminaron por explotar y socavar temporalmente su producción barata de bienes tropicales destinadas a la ecología-mundo. El éxito de los colonizadores franceses en la creación de naturalezas capitalizadas en el sur de Vietnam que generaron productos tropicales baratos para el mundo ecológico estuvo, por tanto, lejos de ser absoluto y carente de contradicciones socioecológicas internas.

\subsection{Conclusión}

El estudio comparativo de la agricultura colonial argelina y las plantaciones de caucho vietnamitas muestra una capacidad desigual de los colonizadores franceses para crear naturalezas capitalizadas que generen productos tropicales baratos. La comparación de las plantaciones vietnamitas con las de Argelia demuestra las capacidades limitadas de los poderes-conocimiento coloniales, siempre dependientes de las ecologías locales, para transformar la naturaleza planificada en naturaleza capitalizada que genera productos tropicales baratos. Por otro lado, el análisis conjunto del desarrollo de la agricultura colonial argelina y las plantaciones vietnamitas revela tanto su capacidad de transformación ecológica del territorio por razones económicas como sus contradicciones internas, ya sean económicas en lo que al caso se refiere. Argelina o socio-ecológico en lo que respecta al caso vietnamita. Esto demuestra, en cualquier caso, que lejos de ser omnipotentes, los colonizadores nunca logran hacer coincidir sus naturalezas planificadas con las realmente existentes, aunque a menudo logran obtener ganancias sustanciales de ellas con la ayuda de una mano de trabajo barato, y así participar. en el mantenimiento del orden colonial. Sin embargo, las plantaciones vietnamitas se distinguen por un éxito económico casi constante, producciones baratas destinadas la ecología-mundo y una importante capacidad de resiliencia ante sus propias contradicciones socioecológicas, a diferencia de las producciones agrícolas en Argelia que rara vez han sido baratas no estaban destinados al mundo ecológico, sino únicamente al mercado metropolitano y tuvieron que ser llevados a distancia por una metrópoli proteccionista, aunque pudo haberse beneficiado durante un tiempo de una tienda de vinos colonial. 


\section{La explotación de los bosques coloniales: ¿Ecologismo colonial o deforestación capitalista?}

\section{I. Ecologismo colonial y explotación de alcornocales en Argelia}

La apropiación colonial de los bosques argelinos a expensas de las poblaciones colonizadas comenzó en 1830 con la confiscación del dominio público otomano, finalmente transformado en propiedad estatal en I85I, y no se justificó por la idea de una terra nullius y por una asimilación de colonizados a no humanos, sino a través de un discurso de restauración ambiental (Davis, 2007; Ford, 2008). Los colonizadores afirmaron así que habían venido a «restaurar el granero de Roma» después de siglos de degradación ambiental ligada al «pastoreo árabe», justificando la prohibición de esto, pero también de la agricultura de roza y quema y la agricultura de roza y quema colección de madera muerta (Davis, 2007). Este discurso fue obviamente solo ficción sincera (Bourdieu, 2008) de ideólogos coloniales desconectados de las realidades sobre el terreno, ya que en realidad la colonización francesa fue inmediatamente acompañada por la destrucción de árboles, como en el oasis de Zaatcha en 1849 donde decenas de miles de productivas palmeras datileras fueron destruidas tras una victoria francesa (Clancy-Smith, 2016). De facto, la apropiación de los bosques argelinos tenía tres objetivos principales: la explotación de sus recursos, el control de las poblaciones colonizadas y la transformación de la economía de subsistencia en una economía capitalista (Davis, 2007). Así, ya en 1846, el Estado francés otorgó concesiones a largo plazo a los inversores franceses ricos que deseaban explotar los bosques de alcornoques a bajo precio (Ford, 2008). Francia era entonces, debido a su producción de vino, el principal consumidor europeo de tapones de corcho, que entonces se producían principalmente en Cataluña a partir de corchos portugueses y españoles (Vilar, 1934, Pp. 286-287). La Argelia colonial se convirtió en pocas décadas en uno de los principales exportadores mundiales de alcornoque, con una producción anual de 6.600 toneladas a finales del siglo XIX y 45.000 a principios de los años 1930 (Vilar, 1934, Pp. 292-295), causando la mayor parte de la deforestación en Argelia entre 1890 y 1940 (Ford, 2008), en un momento en que la deforestación era uno de los principales vectores del cambio climático (Bonneuil \& Fressoz, 2016). Esta deforestación, que no se ralentizó hasta principios de la década de los treinta debido a una crisis de sobreproducción mundial de corcho (Vilar, 1934, p. 297), sin embargo, se culpó a los colonizados, responsabilizados por sus prácticas «incendiarias» y «nómadas» del declive forestal de Argelia desde la conquista árabe del siglo XII (Davis, 2007). Este discurso se basó notablemente en el mito de una Argelia romana exuberante, en contraste con un árabe argelino deforestado, aunque en realidad el Imperio Romano sobreexplotó los recursos de Argelia (Davis, 2007). Si este discurso pretendía esencialmente justificar la colonización francesa y su explotación de los bosques argelinos, también reflejaba una preocupación real de ciertos ideólogos coloniales, la del imperativo de salvaguardar las condiciones climáticas necesarias para el colonialismo de asentamientos, desde la desertificación, las temperaturas de las tierras altas y las condiciones insalubres. como resultado de la disminución de la cubierta forestal, se creía que amenazaba la aclimatación a Argelia de los colonos europeos (Ford, 2008). Si esta preocupación ideológica se hubiera materializado, habría sido un ejemplo interesante de ambientalismo colonial y el deseo de cambiar el clima por razones coloniales (Ford, 2008). Sin embargo, estos discursos no se tradujeron en ninguna medida concreta para proteger los alcornocales, sino solo medidas punitivas como la ley de 1874 que obligaba a las poblaciones colonizadas a combatir los incendios forestales e imponerles multas colectivas cuando se les consideraba responsables de un incendio 
(Ford, 2008). La única medida concreta del ecologismo colonial fue la creación de trece parques nacionales en 1921 por motivos ideológicos y turísticos, ya que se trataba de mostrar al visitante un "ambiente francés en Argelia" (Ford, 2008). Al final, la apropiación y explotación barata de los bosques argelinos, especialmente el corcho, no se vio obstaculizada en modo alguno por el ecologismo colonial, sino al contrario, justificada por él. El mundo de la ecología se benefició así del corcho barato en nombre de su conservación.

\section{2. Ecologismo colonial y explotación de los bosques vietnamitas}

La colonización de Vietnam tiene muchas similitudes con la de Argelia en cuanto a la apropiación y explotación barata de sus bosques. En este caso, sin embargo, fue la armada francesa la que, para asegurar su suministro de madera, inició la explotación de los bosques del sur de Vietnam recurriendo a mano de obra barata y forzada (McElwee, 2016; Thomas, 2009). Sin embargo, el papel central inicial de la armada francesa en la explotación de los bosques como en la del carbón, pronto dio paso a un proceso similar al de Argelia: una apropiación estatal de los bosques a expensas de las poblaciones locales y su subsistencia. explotación de concesiones forestales que conducen a una intensa deforestación (McElwee, 2016;Thomas, 2009).Así, en I866, el gobernador de Cochinchina autorizó por motivos fiscales la explotación comercial de los bosques vietnamitas, lo que aportaría dinero al presupuesto colonial a través de impuestos, permisos y concesiones (McElwee, 2016;Thomas, 2009). Esta privatización de los bosques se llevó a cabo en detrimento de la población local, que veía cada vez más limitado su acceso a los recursos forestales, condicionado a jornadas de trabajo forzoso y con castigos colectivos en caso de tala no autorizada (McElwee, 2016; Thomas, 2009). Esta situación se agravó con el tiempo con impuestos cada vez más altos y nuevas restricciones (McElwee, 20 I6;Thomas, 2009).Así, a pesar de que la creciente deforestación de los bosques vietnamitas tuvo lugar inicialmente de forma concéntrica alrededor de Saigón, la capital colonial, se atribuyó, como en Argelia, sin ninguna prueba a las prácticas de los colonizados, en particular la agricultura de roza y quema, justificando a los ojos de la administración colonial un completo control forestal (McElwee, 2016; Thomas, 2009). La administración forestal, lejos de ser una élite ilustrada y de mente abierta (Grove, 1997), persistió en culpar a las prácticas de los colonizados de arcaicas, bárbaras e irracionales, que debían ser sustituidas por un modo de explotación más racional y científico (McElwee, 2016;Thomas, 2009), a pesar de que estas prácticas eran esenciales para la forma de vida de las poblaciones locales, económicamente racionales y no ecológicamente destructivas (Aso, 20l8). Este paternalismo forestal colonial no tuvo en un principio otro resultado que lavar a las empresas coloniales de sus responsabilidades, sin que se tomaran medidas de protección (McElwee, 2016; Thomas, 2009). Sin embargo, la deforestación colonial creció hasta tal punto que obligó a las autoridades coloniales en I89I a crear reservas forestales, en las que se debían aplicar métodos de tala sostenible (McElwee, 2016; Thomas, 2009). No obstante, la tala barata continuó sin obstáculos en todas partes y, en realidad, la mayoría de las reservas forestales estaban prácticamente protegidas, o ya estaban deforestadas o tenían recursos forestales comercialmente inutilizables (McElwee, 2016; Thomas, 2009). El ecologismo colonial de la administración forestal fue en gran parte ineficaz, y cuando se dignó oponerse a la tala ilegal de la segunda mina de carbón más grande de Vietnam, el asunto se prolongó durante una década antes. Para terminar en una victoria para la empresa minera debido a las reducciones de personal. en la administración forestal tras la crisis de los años 1930 (Campagne, 2021). Finalmente, como explica 
Frédéric Thomas (2009), las prácticas de tala sostenible vietnamitas eran relativamente marginales, y la ideología forestal conservacionista siguió siendo una ideología que justifica la exclusión de las poblaciones locales del acceso a los recursos forestales. El principal obstáculo para la tala colonial siguió siendo la falta de interés económico, la (demasiado) gran biodiversidad, el lento crecimiento de los árboles y la inaccesibilidad de muchos bosques. (McElwee, 20I6).

La prioridad siguió siendo la explotación comercial barata de los recursos forestales de Vietnam, en particular para permitir la expansión de las redes ferroviarias y telegráficas (McElwee, 2016; Thomas, 2009), sino también para la exportación a la ecología-mundo y particularmente a China. Así, los servicios forestales coloniales terminaron poniéndose de lleno al servicio de la producción de madera barata, clasificando los bosques según su interés económico y no por criterios ecológicos, concentrando su presencia en bosques de fácil acceso y con alto valor agregado, y poniendo inundaciones y prevención de deslizamientos de tierra detrás de los intereses capitalistas forestales (McElwee, 2016; Thomas, 2009). A mediados de los años treinta, finalmente, incluso las reservas forestales se abrieron a la explotación capitalista insostenible, acompañada de fraude y derroche sistemático (McElwee, 2016; Thomas, 2009). Esto resultó en una deforestación masiva, con 12 millones de hectáreas de bosques fuertemente deforestados en 1933, sin mencionar su pérdida en términos de biodiversidad (McElwee, 2016;Thomas, 2009). Esta situación resultó, en particular, del continuo cabildeo de las empresas agrícolas y mineras coloniales, que se opusieron a las reservas forestales y en ocasiones obtuvieron su desclasificación (McElwee, 2016; Thomas, 2009).Y cuando este no fue el caso, las empresas carboneras explotaron ilegalmente los bosques circundantes para asegurar su suministro de puntales mineros, hasta que en 1925 el Gobernador General de Indochina autorizó parcialmente la explotación comercial de las reservas forestales, abandonando de paso el principio de explotación sostenible. que los servicios forestales estaban tratando de establecer (McElwee, 2016; Thomas, 2009). Paradójicamente, las poblaciones locales fueron más respetuosas con el código forestal que las empresas capitalistas coloniales, y esto aunque nunca dejaron de desplegar las artes de resistencia destacadas por James C. Scott (2000), a veces llegando incluso a la violencia e incluso a la insurrección armada como en 1930-3 I (McElwee, 20 I 6; Thomas, 2009). Por lo tanto, las regiones forestales fueron particularmente hostiles al colonialismo francés. Al final, el ecologismo colonial de la administración forestal nunca fue un obstáculo real para la explotación barata de los recursos forestales vietnamitas. Solo justificó la apropiación colonial de los bosques a expensas de las poblaciones locales sin limitar la extensión de la deforestación de origen colonial.

\section{3. Conclusión}

El estudio comparativo de la explotación barata de los recursos forestales por empresas coloniales en Vietnam y Argelia con miras en particular a ser exportados a la ecología-mundo muestra hasta qué punto el ecologismo colonial francés no fue un obstáculo para esto primero, sino al contrario. Justificó la apropiación colonial de los bosques vietnamitas y argelinos a expensas de las poblaciones locales. La similitud de los casos vietnamita y argelino en términos de represión paternalista de las prácticas de los colonizados se explica en particular por una matriz común, la de la apropiación violenta por parte del Estado francés de los bosques de Francia a expensas de las poblaciones locales del antiguo régimen. Sin embargo, la ideología ecológica que justificaba 
la apropiación de los recursos forestales tomó no solo una forma específica de las colonias, sino también dentro de cada colonia, de ahí en particular la ausencia de parques nacionales en el Vietnam colonial, donde no existía naturaleza francesa (es decir, mediterránea) a ser protegida de una manera relativamente imperativa. Además, al no ser la colonización de Vietnam una colonización de colonos, a diferencia de la de Argelia, los ideólogos coloniales de Vietnam no temían la degeneración de los colonos europeos por motivos medioambientales. Sin embargo, estas diferencias ideológicas tuvieron poco impacto concreto en términos de deforestación. Más significativamente, la explotación barata de los bosques vietnamitas y argelinos formaba parte de la ecología-mundo de una manera diferente, ya que el producto estrella de este último (el corcho) se enviaba principalmente a Francia, mientras que la mayor parte de la madera vietnamita exportada se dirigía a China. Esta diferencia en el registro en la ecología mundial de la producción forestal de Argelia y Vietnam se hace eco de la producción de combustibles fósiles (aparte del carbón argelino), y esto también principalmente por razones geográficas. Pero en cada caso, la apropiación y explotación de los recursos forestales fue barata y no se vio obstaculizada por el ecologismo colonial, más bien, al contrario.

\section{Conclusión}

El estudio histórico de los combustibles fósiles, bosques y plantaciones de Argelia (1830-1962) y Vietnam (1858-1954) en términos de ecología-mundo muestra que sí es esclarecedor estudiarlos a través de tal marco conceptual, su contribución al desarrollo de la ecología mundial debe examinarse a la luz de las especificidades de cada colonia y de cada sector. En primer lugar, debe matizarse la hipótesis de una producción colonial de combustibles fósiles que sería sistemáticamente barata y destinada a los centros capitalistas occidentales de la ecología-mundo, no correspondiendo esta hipótesis ni al carbón argelino (caro y usado localmente), ni a los hidrocarburos argelinos (caros y exportados principalmente a Francia) ni carbón vietnamita (barato pero exportado al sudeste asiático). Luego, la historia conjunta de las plantaciones de caucho vietnamitas y la agricultura colonial argelina revela una capacidad desigual y nunca absoluta de los colonizadores franceses para crear naturalezas capitalizadas generando productos tropicales baratos. Finalmente, el estudio combinado de la explotación colonial de los recursos forestales de Argelia y Vietnam permite mostrar en qué medida el ecologismo colonial francés no obstaculizó las producciones forestales coloniales baratas destinadas a la ecología-mundo. Este análisis, evidentemente, merecería ser profundizado y matizado por nuevos estudios de caso, pero constituye, sin embargo, un primer vistazo a la compleja inserción histórica de las colonias francesas en la ecología-mundo.

\section{Referencias}

Aso, M. (2018). Rubber and the making of Vietnam:An ecological history, I897-1975. The University of North Carolina Press.

Bonneuil, C. (1997). Mettre en ordre et discipliner les tropiques : Les sciences du végétal dans l'Empire français, I870-I940. Paris VII.

Bonneuil, C., \& Fressoz, J.-B. (2016). L'événement anthropocène. Seuil.

Bouchène, A., Peyroulou, J.-P., Siari Tengour, O., \& Thénault, S. (Eds.). (20I4). Histoire de l’Algérie à la période coloniale, 1830-1962. Éd. La Découverte.

Bourdieu, P. (2008). The logic of practice. Stanford Univ. Press.

Brocheux, P., \& Hémery, D. (20I I). Indochina:An Ambiguous Colonization, I858- 1954. University of California Press. 
Campagne, A. (20I7a). Histoire des productions industrielles de charbon en Indochine (I873-1955) [Mémoire de Master 2]. EHESS.

Campagne,A. (20I7b). Le Capitalocène.Aux racines historiques du dérèglement climatique. Éditions Divergences.

Campagne, A. (2020). Coal, French energy imperialism and the conquest of Vietnam (1873-I885). Journal of Energy History/Revue d'Histoire de l'Énergie, 3. http://energyhistory.eu/node/2I8

Campagne, A. (202I). French colonialism and coal mining in Algeria and Vietnam, I873-1962. Institut Européen Universitaire.

Cantoni, R. (2017). Oil Exploration, Diplomacy, and Security in the Early Cold War:The Enemy Underground. Routledge.

Clancy-Smith, J. (2016). L'Afrique du Nord à l'époque coloniale : Migration, agriculture et échec de l'innovation, I83019|4. Revue d'histoire Du XIXe Siècle, 53, 97-I|4.

Cooper, F., \& Stoler, A. L. (Eds.). ( 1997). Tensions of empire: Colonial cultures in a bourgeois world. University of California Press.

Crosby, A.W. (2003). The Columbian exchange: Biological and cultural consequences of I 492. Praeger.

Davis, D. K. (2007). Resurrecting the granary of Rome: Environmental history and French colonial expansion in North Africa. Ohio University Press.

Fichter, J. R. (20I2). British Infrastructure and French Empire:Anglo-French Steam Interdependency in Asian Waters, c. 1852-1870. Britain and the World, 5(2), I83-203.

Fichter, J. R. (2019). Imperial Interdependence on Indochina's Maritime Periphery: France and Coal in Ceylon, Singapore, and Hong Kong, I859-I895. In J. R. Fichter (Ed.), British and French Colonialism in Africa, Asia and the Middle East (pp. I5I-179). Springer International Publishing.

Ford, C. (2008). Reforestation, Landscape Conservation, and the Anxieties of Empire in French Colonial Algeria. The American Historical Review, I I3(2), 34I-362.

Frémeaux, J. (20I0). Le Sahara et la France. SOTECA.

Garrier, G. (1989). Le phylloxéra : Une guerre de trente ans, I870-/ 900.A. Michel.

Grove, R. H. (1997). Green imperialism: Colonial expansion, tropical island Edens and the origins of environmentalism, I600 - 1860. Cambridge Univ. Press.

Hamilton, P. E. (2020). The Imperial and Transpacific Origins of Chinese Capitalism. Journal of Historical Sociology, 33(I), 134-148.

Henni, A. (2020). Économie de l'Algérie coloniale, I830-I 954.

Isnard, H. (1949).Vigne et colonisation en Algérie. Annales de Géographie, 58(3 I I), 2I 2-2 I 9.

Jaehyun, J. (2018). Exploitation minière et exploitation humaine : Les charbonnages dans le Vietnam colonial, I874-1945 [Thèse de doctorat en Histoire]. Université Paris Diderot - Paris 7.

Larab, F. (2013). Les mineurs du désert : Kenadsa 19/3-1962.

Malm,A. (2016a). Fossil Capital:The Rise of Steam Power and the Roots of Global Warming.Verso.

Malm, A. (2016b). Who Lit This Fire? Approaching the History of the Fossil Economy. Critical Historical Studies, 3(2), $215-248$.

Marseille, J. (2005). Empire colonial et capitalisme français : Histoire d'un divorce. A. Michel.

McElwee, P. D. (20I6). Forests are gold:Trees, people, and environmental rule in Vietnam. University of Washington Press.

Meynier, G. (2015). L'Algérie révélée : La guerre de 19/4 - 19/8 et le premier quart du XXe siècle. Bouchène Editions.

Moore, J. (2007). Ecology and the Rise of Capitalism, PhD Dissertation, Department of Geography. University of California.

Moore, J. (2015). Capitalism in the Web of Life: Ecology and the Accumulation of Capital.Verso.

Murray, M. J. (1980). The development of capitalism in colonial Indochina (I870-1940). University of California Press.

Patel, R., \& Moore, J.W. (2017). A history of the world in seven cheap things: Guide to capitalism, nature, and the future of the planet. Oakland, California.

Pomeranz, K. (2002). Political Economy and Ecology on the Eve of Industrialization: Europe, China, and the Global Conjuncture. The American Historical Review.

Saul, S. (2016). Intérêts économiques français et décolonisation de l'Afrique du Nord. Librairie Droz.

Scott, J. C. (2000). Weapons of the weak: Everyday forms of peasant resistance. Yale Univ. Press.

Sessions, J. E. (20I4). By sword and plow: France and the Conquest of Algeria. Cornell University Press.

Thomas, F. (2009). Protection des forêts et environnementalisme colonial : Indochine, 1860-1945. Revue d'histoire moderne et contemporaine, 56-4 (4), 104.

Touati, I. (20I8). Le commerce du blé entre l'Algérie et la France, XVle-XIXe siècles. Éditions Bouchène.

Tucker, R. P., \& Richards, J. F. (Eds.). ( 1 983). Global deforestation and the nineteenth-century world economy. Duke University Press.

Vilar, P. (I 934). L’Espagne et le commerce mondial du liège. Annales de Géographie, 43(243), 282-298. 


\section{RELACIONES INTERNACIONALES}

Revista académica cuatrimestral de publicación electrónica

Grupo de Estudios de Relaciones Internacionales (GERI)

Universidad Autónoma de Madrid, España

https://revistas.uam.es/relacionesinternacionales

ISSN 1699 - 3950

f facebook.com/RelacionesInternacionales

3. twitter.com/RRInternacional 